\title{
SHORT-TERM ELECTROMAGNETIC PRECURSORS OF EARTHQUAKES
}

\author{
B. V. Dovbnya1, A. Yu. Pashinin², R. A. Rakhmatulin² \\ ${ }^{1}$ Borok Geophysical Observatory of O.Yu. Schmidt Institute of Physics of the Earth of RAS, \\ Borok, Yaroslavl region, Russia \\ 2 Institute of Solar-Terrestrial Physics, Siberian Branch of RAS, Irkutsk, Russia
}

\begin{abstract}
In the study of the relationship between electromagnetic and seismotectonic processes, we analyzed the data of three observatories located at large distances, considering both latitudes and longitudes, and detected electromagnetic impulses in the frequency range from 0 to $5 \mathrm{~Hz}$, which were recorded minutes before earthquakes. Detailed morphological and statistical analysis was carried out, and common and specific features were considered. Several cases are discussed to illustrate the occurrence of electromagnetic precursor signals before the recorded devastating earthquakes in Japan, China, Romania and other seismically active regions. A qualitative explanation is given for the study results.
\end{abstract}

Key words: electromagnetic impulse; earthquake; precursor 


\title{
КРАТКОСРОЧНЫЕ ЭЛЕКТРОМАГНИТНЫЕ ПРЕДВЕСТНИКИ ЗЕМЛЕТРЯСЕНИЙ
}

\author{
Б. В. Довбня1', А. Ю. Пашинин², Р. А. Рахматулин ${ }^{2}$ \\ ${ }^{1}$ Геофизическая обсерватория «Борок» Института физики Земли им. О.Ю. Шмидта РАН, \\ п. Борок, Ярославская область, Россия \\ ${ }^{2}$ Институт солнечно-земной физики СО РАН, Иркутск, Россия
}

\begin{abstract}
Аннотация: При исследовании связи электромагнитных и сейсмотектонических процессов по данным трех разнесенных по широте и долготе обсерваторий обнаружены электромагнитные импульсы в диапазоне частот от 0 до 5 Гц, которые наблюдались за минуты до землетрясения. Проведен детальный морфологический и статистический анализ результатов, рассмотрены их общие и индивидуальные особенности. На конкретных примерах иллюстрируется появление сигналов перед разрушительными землетрясениями в Японии, Китае, Румынии и в ряде других сейсмоактивных регионов. Обсуждаются затронутые в работе проблемы, полученным результатам дается качественное объяснение.
\end{abstract}

Ключевые слова: электромагнитный импульс; землетрясение; предвестник

\section{1. ВВЕДЕНИЕ}

В последние десятилетия по данным спутниковых и наземных наблюдений накоплен большой экспериментальный материал о разнообразных проявлениях сейсмической активности в верхней атмосфере и переменном электромагнитном поле Земли (см., например, монографии В.А. Липеровского [Liperovsky et al., 1992], Г.А. Соболева [Sobolev, 1993] и М. Хаякавы [Hayakawa, 2001], обзор [Guglielmi, 2007]). Ряд работ посвящен исследованию связи землетрясений с магнитными бурями, имеющими внезапное начало (SSC). Обнаружено изменение сейсмической активности через несколько дней после SSC [Sobolev et al., 2001; Zakrzhevskaya, Sobolev, 2002, 2004; Adushkin et al., 2012].

Другое направление, к которому и относится предлагаемая здесь работа, связано с изучением электромагнитных явлений, предваряющих и сопровождающих землетрясения. Накопленный к настоящему времени экспериментальный материал указывает на перспективность исследований таких явлений в ультранизкочастотном (УНЧ) диапазоне (0.001-10.000 Гц) [Ismaguilov et al., 2001].

К первым наблюдениям относятся работы [Kopytenko et al., 1993; Molchanov, 1990; Molchanov et al., 1992], в которых сообщается о колебаниях геомагнитного поля перед разрушительным землетрясением в Спитаке. Также следует отметить работы [Fraser-Smith et al., 1990; Bernardi et al., 1991], в которых обнаружен и проанализирован мощный всплеск ультранизкочастотных электромагнитных колеба- ний перед землетрясением в Лома-Приета. В работе [Hayakawa et al., 1996] сообщается о наблюдении сверхнизкочастотного электромагнитного шума перед землетрясением в Гуаме. Обнаружены УНЧ геомагнитные возмущения, отражающие процесс подготовки Суматра-Андаманского землетрясения [Sobisevich et al., 2010; Sobisevich L.E., Sobisevich A.L., 2010].

Таким образом, имеющиеся к настоящему времени отдельные наблюдения указывают на возможность возникновения перед сейсмическими событиями электромагнитных явлений в УНЧ диапазоне. И все же, как полагают авторы работ [Kosterin et al., 2015; Thomas et al., 2009a, 2009b], результаты разрозненных наблюдений нельзя еще считать строгим доказательством связи УНЧ эмиссий с землетрясениями.

В геофизической обсерватории «Борок» была предпринята попытка по данным непрерывных записей УНЧ вариаций электромагнитного поля Земли исследовать связь электромагнитных и сейсмотектонических процессов. В результате удалось обнаружить специфические УНЧ электромагнитные импульсы в диапазоне частот 0-5 Гц, наблюдаемые в выделенной временно́й окрестности землетрясений [Dovbnya et al., 2006]. Особое внимание привлекли те из них, которые наблюдались до сейсмического события. Появление упреждающих сигналов представляло несомненный интерес в рамках актуальной проблемы геофизики - поиска предвестников землетрясений (см., например [Hayakawa, 1999; Hayakawa, Molchanov, 2002; Hattori, 2004; Guglielmi, Zotov, 2012]). 
Изучению этого вопроса и посвящена настоящая работа. На основе тщательного анализа большого массива геофизических данных в трех разнесенных по широте и долготе обсерваториях мы приведем экспериментальные факты, свидетельствующие о появлении электромагнитных предвестников, и обсудим полученные результаты.

\section{2. ИСХОДНЫЙ МАТЕРИАЛ И МЕТОДЫ АНАЛИЗА}

Анализ ультранизкочастотных излучений выполнялся по данным магнитных измерений, полученных двумя среднеширотными обсерваториями - Геофизической обсерваторией «Борок» Института физики Земли РАН (58.1 ${ }^{\circ}$ с.ш., 38.2 ${ }^{\circ}$ в.д.) за период с 1973 по 1995 г. и обсерваторией «Монды», расположенной на территории Саянской солнечной обсерватории Института солнечно-земной физики СО РАН (51.6º с.ш., $100.9^{\circ}$ в.д.) за период с 2011 по 2015 г., а также высокоширотной Геофизической обсерваторией «Колледж» (64.9 с.ш., $148.0^{\circ}$ 3.д.), расположенной в штате Аляска, США, за период с 1973 по 1977 г. Исходным материалом для анализа являлись записи УНЧ вариаций электромагнитного поля Земли. В обсерваториях «Борок» и «Колледж» для измерений использовался индукционный магнитометр с регистрацией на аналоговый магнитофон, в обсерватории «Монды» - цифровой индукционный магнитометр LEMI-30. Во всех обсерваториях регистрировалось по две горизонтальные компоненты магнитных вариаций, север-юг и восток-запад. Амплитудно-частотная характеристика приборов позволяла анализировать колебания с частотой до 10 Гц в обсерваториях «Борок»и «Колледж»и до 30 Гц в обсерватории «Монды». Аналоговые записи, полученные в обсерваториях «Борок» и «Колледж», оцифровывались, а затем подвергались спектрально-временному анализу с применением компьютерных программ так же, как и цифровые записи обсерватории «Монды». Строились динамические спектры колебаний (спектрограммы), на которых в координатах частота-время отражалась информация о переменном электромагнитном поле в анализируемом интервале. При первоначальном визуальном просмотре из дальнейшего анализа исключались известные формы сигналов магнитосферного происхождения. Сигналы, не коррелирующие с другими геофизическими явлениями, сопоставлялись с данными каталога землетрясений (International Seismological Centre, ISC Catalogues, https://www.isc.ac.uk). За момент появления сигнала бралась середина длительности импульса, которая определялась по временной шкале спектрограммы с точностью до 10 с.

\section{3. РЕЗУЛЬТАТЫ НАБЛЮДЕНИЯ}

Примерно для 250 событий (землетрясений) со статистической значимостью $\mathrm{p}=0.86$ привязки к ближайшему по каталогу землетрясению были зарегистрированы сигналы, наблюдаемые в первые десятки секунд или минуты до сейсмического события. По виду динамического спектра это были либо единичные, либо парные электромагнитные импульсы в диапазоне частот от 0 до 5 Гц. Амплитуда сигнала обычно не превышала значения 10 пТл, а длительность - 20 с. При парном проявлении время между первым и вторым импульсами варьировалось в основном в интервале от 10 до 40 с. Реже наблюдались серии из трех и более импульсов. Как правило, динамические спектры импульсов имели дискретную структуру. На рис. 1 даны распределения единичных (1i) и парных (2i) импульсов, построенные по результатам наблюдений в трех обсерваториях. На верхней панели рисунка распределения приведены в интервале 0-11 мин до землетрясения, на нижней - за минуту. Из рисунка видно, что в основном перед землетрясением наблюдались единичные импульсы. Отдельные сигналы регистрировались уже за 10 мин, но все же большая их часть появлялась ближе к моменту землетрясения. За минуту до землетрясения электромагнитная активность резко падала, и в последние 20 с импульсы не были замечены. Примерно такой же характер наблюдался и в распределении парных импульсов.

Вероятность появления сигналов в отдельной обсерватории при одинаковой в среднем сейсмической активности оказалась существенно неодинаковой в разные дни и даже недели. Периоды их полного отсутствия вдруг сменялись периодами неожиданно высокой электромагнитной активности. Сигналы после затишья начинали появляться, при этом вероятность их наблюдения не зависела существенно от магнитуды события, но отмечалось предпочтение для близких (2-3 тыс. км) землетрясений.

Электромагнитные предвестники были зарегистрированы перед разрушительными землетрясениями в Японии, Китае, Румынии, перед землетрясениями в Турции и других сейсмоактивных регионах. Примеры предвестников обоих типов даны на рис. 2, a-8. Здесь и далее темными треугольниками отмечены моменты землетрясений, стрелками - их предвестники, светлыми треугольниками отмечается приход сейсмической волны. В подписях к рисункам приводятся следующие параметры землетрясений: мировое время, географические координаты, глубина h в км, магнитуда М. На панели (a) рассмотрен электромагнитный отклик на событие в Румынии. Разрушительное землетрясение с 

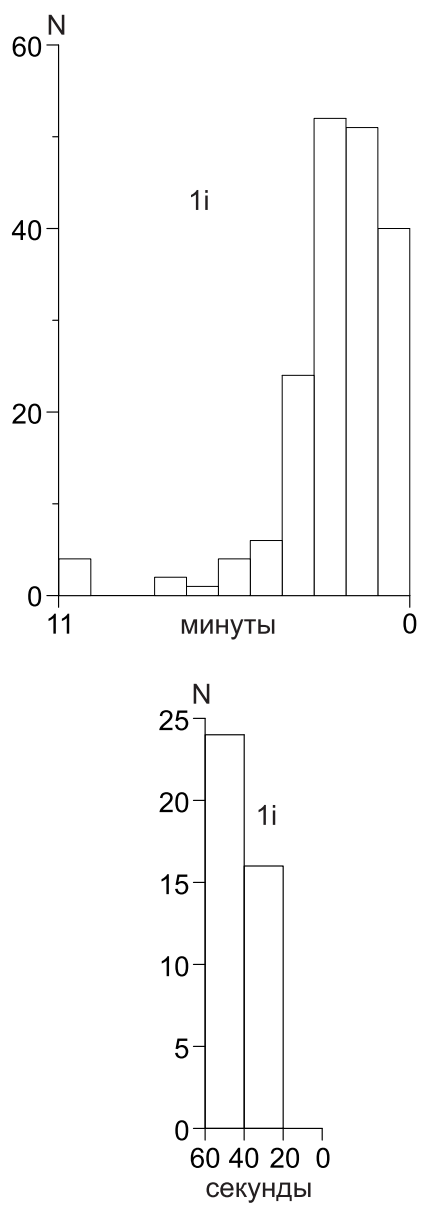
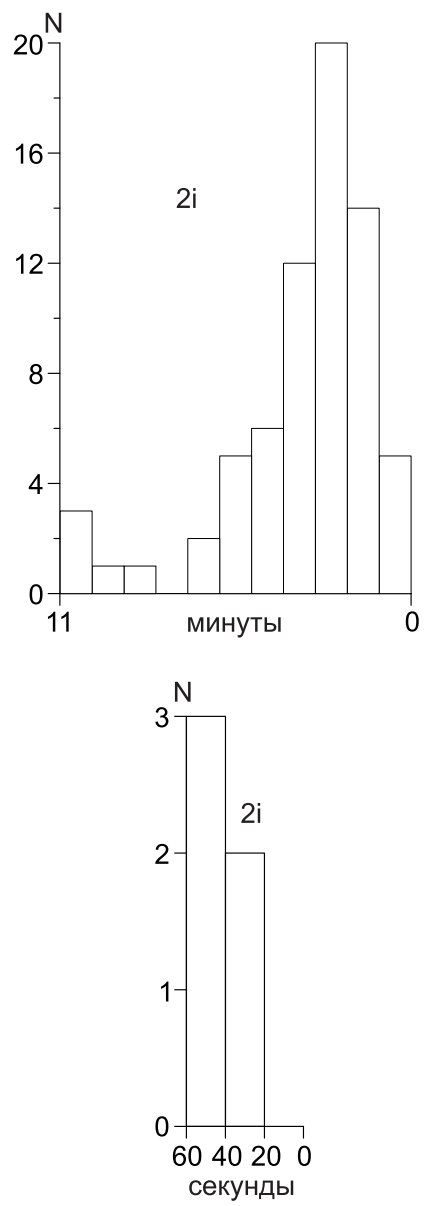

Рис. 1. Распределение электромагнитных сигналовпредвестников в интервале 0-11 мин (верхняя панель) и за минуту до землетрясения (нижняя панель). Слева - одиночные сигналы, справа - парные.

Fig. 1. Distribution of electromagnetic precursor signals in the range of $0-11$ min (upper panel) and one minute before the earthquake (lower panel). Left - single signals; right - paired signals.
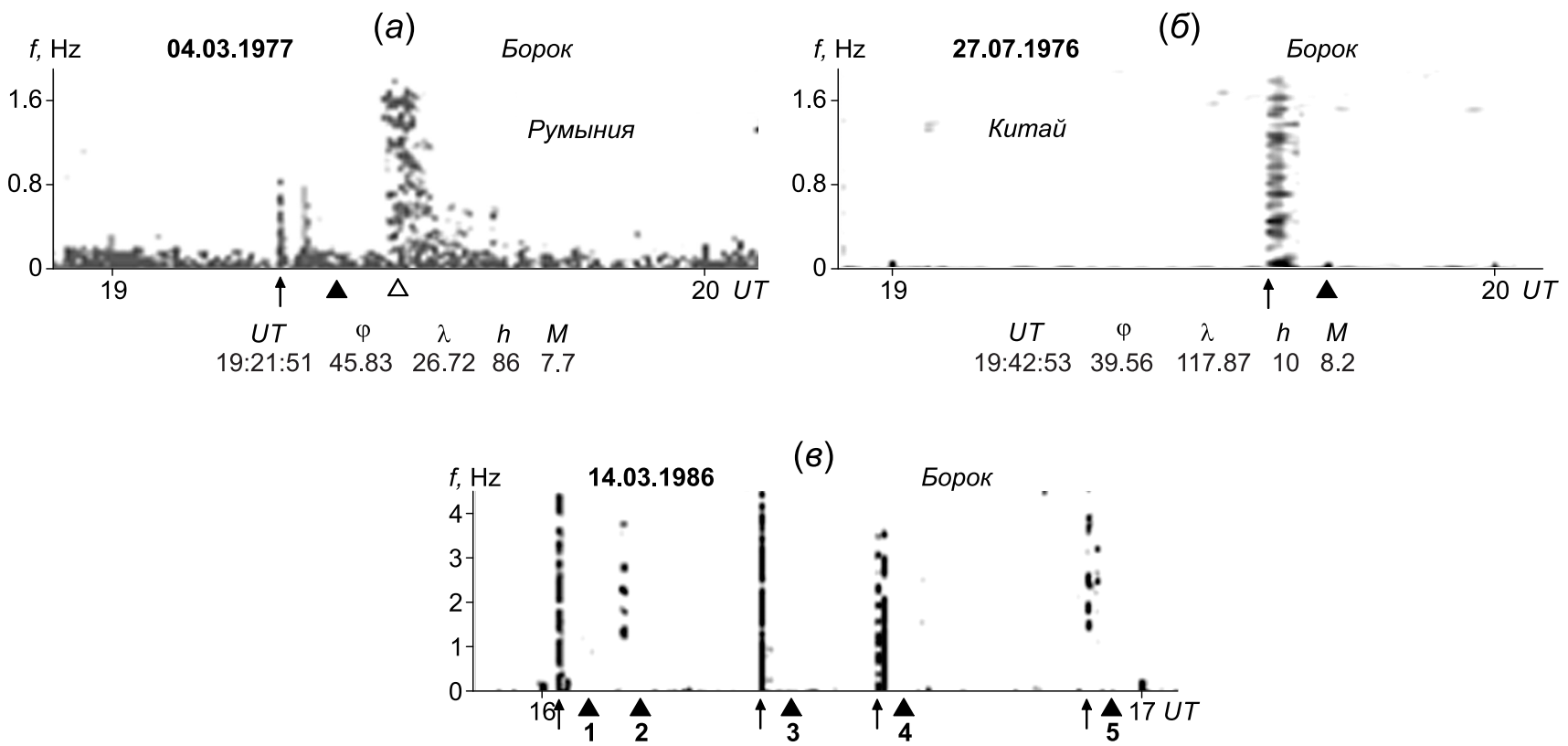

$(8)$

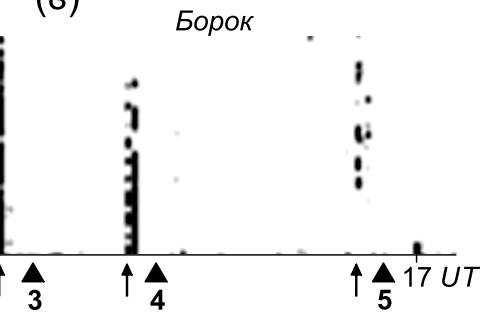

\begin{tabular}{crrrr}
$U T$ & $\varphi$ & \multicolumn{1}{c}{$\lambda$} & \multicolumn{1}{c}{$h$} & $M$ \\
$\mathbf{1}-16: 06: 13$ & -5.28 & 154.40 & 233 & 5.2 \\
$\mathbf{2}-16: 09: 49$ & 38.84 & 144.50 & 20 & 3.7 \\
$\mathbf{5}-16: 55: 52$ & -30.10 & -176.50 & 33 & 6.5
\end{tabular}

Рис. 2. Примеры краткосрочных предвестников землетрясений.

Fig. 2. Examples of short-term precursors of earthquakes. 

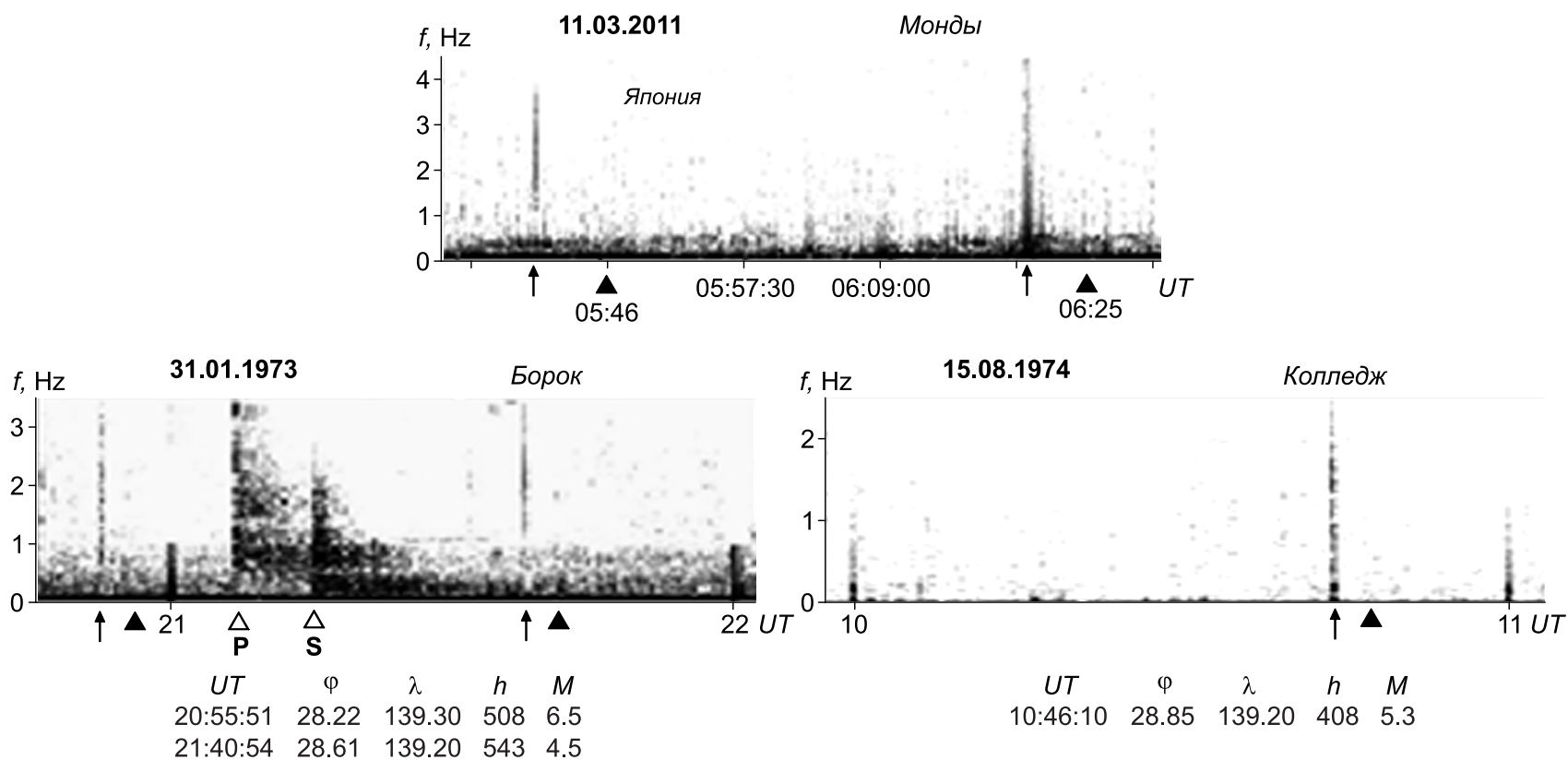

Рис. 3. Наблюдение предвестников землетрясений в Японии по данным обсерваторий «Монды», «Борок» и «Колледж».

I Fig. 3. Observed precursors of earthquakes that occurred in Japan. Data of the Mondy, Borok and College Observatories.

магнитудой M=7.7 произошло 4 марта 1977 г. в 19:21 UT. За четыре минуты до момента землетрясения в обсерватории «Борок» была зарегистрирована пара электромагнитных импульсов, которые на 10 мин опередили приход сейсмической волны из эпицентра главного удара (отмечен светлым треугольником на рисунке). 27 июля 1976 г. в Китае произошло катастрофическое землетрясение с $\mathrm{M}=8.2$. За четыре минуты до момента землетрясения был также зарегистрирован его предвестник (рис. 2, б). На панели (в) даны примеры предвестников землетрясений, произошедших в разных регионах и с разной магнитудой.

На рис. 3 рассмотрены случаи появления электромагнитных сигналов перед землетрясениями в Японии по наблюдениям в трех разных обсерваториях. 11 марта 2011 г. у берегов Японии в 05:46 UT произошло самое мощное за всю историю наблюдений землетрясение. Его магнитуда составила 9.1 по шкале Рихтера. Землетрясение сопровождалось серией повторных толчков, наиболее сильный из них с M=7.2 зафиксирован в 06:25 UT. На верхней панели рис. 3 это событие рассматривается по данным магнитных измерений в обсерватории «Монды». Здесь по горизонтальной оси нанесены метки времени с интервалом 11.5 мин между ними. За 5 мин до главного толчка в обсерватории регистрируется электромагнитный импульс. Перед повторным толчком наблюдается более интенсивный сигнал, который также опережает момент землетрясения на 4 мин. На нижней панели рис. 3 даны примеры регистрации предвестников в «Бороке» и «Колледже». Рассмотрим рисунок слева. У берегов Японии 31 января произошло два глубокофокусных землетрясения с близко расположенными эпицентрами. Их магнитуды М составили 6.5 и 4.5 соответственно. На спектрограмме моменты землетрясений отмечены темными треугольниками. Как и в случае 11 марта 2011 г., перед каждым из сейсмических событий мы видим электромагнитные импульсы (показаны стрелками). Опережение в данном случае составило около 5 мин. В «Бороке» снова отмечается приход сейсмических волн из эпицентра землетрясения. Справа на панели дан пример регистрации импульса-предвестника землетрясения в Японии с $\mathrm{M}=5.3$ по наблюдениям в обсерватории «Колледж». Опережение составило около 5 мин.

На рис. 2 и 3 можно заметить примечательное свойство проявления электромагнитной активности - повторяемость предвестников в землетрясениях, происходящих вслед за главным ударом в том же регионе. Эта особенность представляет отдельный интерес, мы проиллюстрируем ее примерами на рис. 4 (подробно повторяемость рассматривается в работе [Dovbnya, 2014]), на котором моменты землетрясений отмечены черными треугольниками, их предвестники отмечаются стрелками.

На рисунке 5 дан пример одновременного появления электромагнитного сигнала-предвестника в обсерваториях «Борок» и «Колледж». В обеих обсерваториях сигналы имеют одинаковую спектраль- 

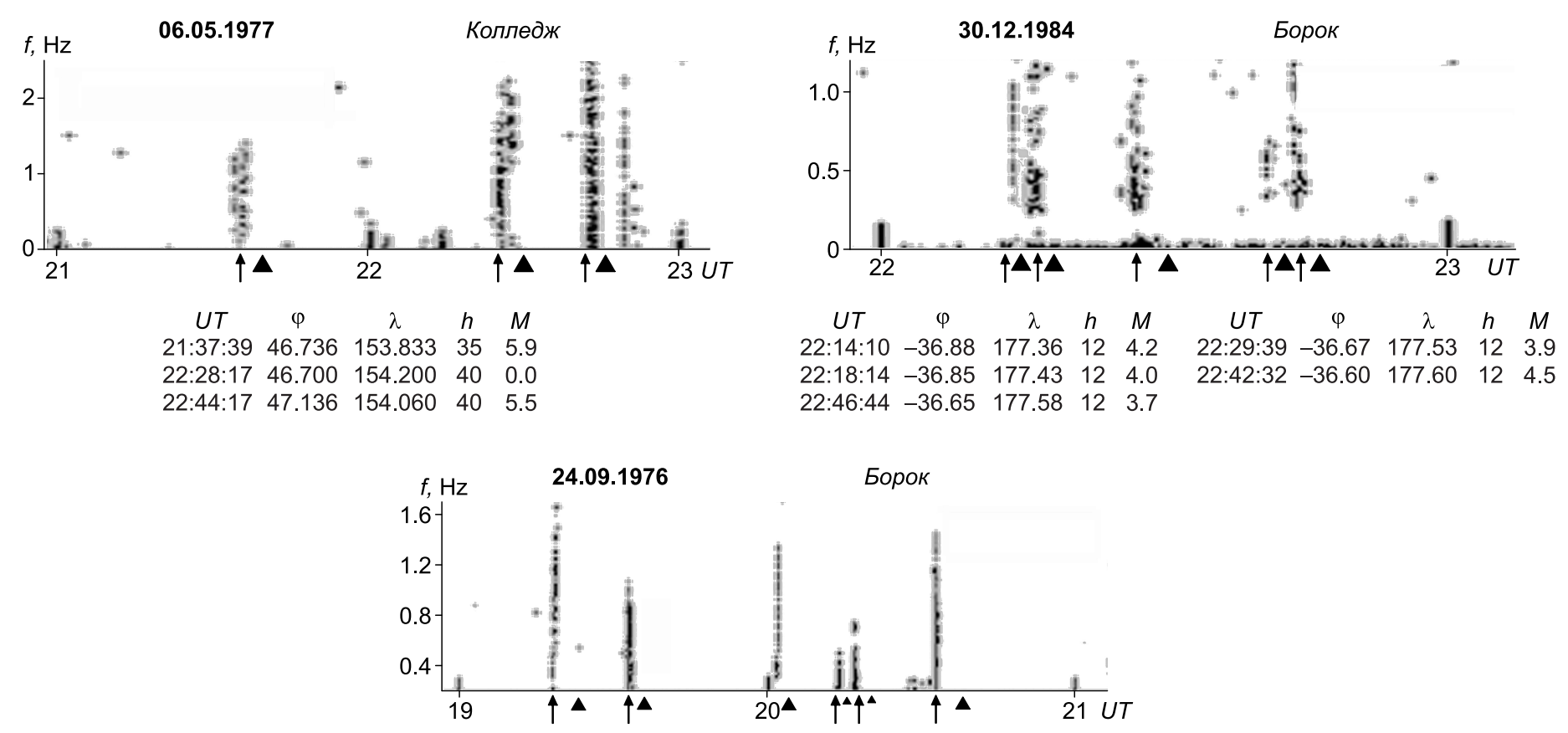

$\begin{array}{cccccccccc}U T & \varphi & \lambda & h & M & U T & \varphi & \lambda & h & M \\ \text { 19:23:27 } & 38.86 & 26.76 & 0 & 3.3 & 20: 15: 20 & 38.370 & 26.800 & 0 & 2.9 \\ \text { 19:30:44 } & 38.66 & 26.25 & 0 & 2.9 & 20: 17: 51 & 38.314 & 26.797 & 10 & 3.3 \\ \text { 20:03:29 } & 38.41 & 26.72 & 11 & 4.1 & 20: 38: 57 & 38.139 & 26.387 & 6 & 3.7\end{array}$

Рис. 4. Примеры повторяемости предвестников.

Fig. 4. Examples showing the recurrence of precursors.

ную форму и наблюдаются практически одновременно, несмотря на то, что они почти на 12 часов разнесены по долготе и на 10 градусов по широте.

Иногда перед землетрясениями могут наблюдаться серии из следующих друг за другом с разными интервалами импульсов. Число импульсов в серии может меняться от 3 до 10, интервалы между ними - от 1 до 2 мин, длительность серий может доходить до 15 мин, хотя в основном она составляет 5-10 мин. Спектры таких серий-предвестников представлены на рис. 6.

На этом мы закончим краткий обзор результатов наблюдения краткосрочных предвестников землетрясений и перейдем к их обсуждению.
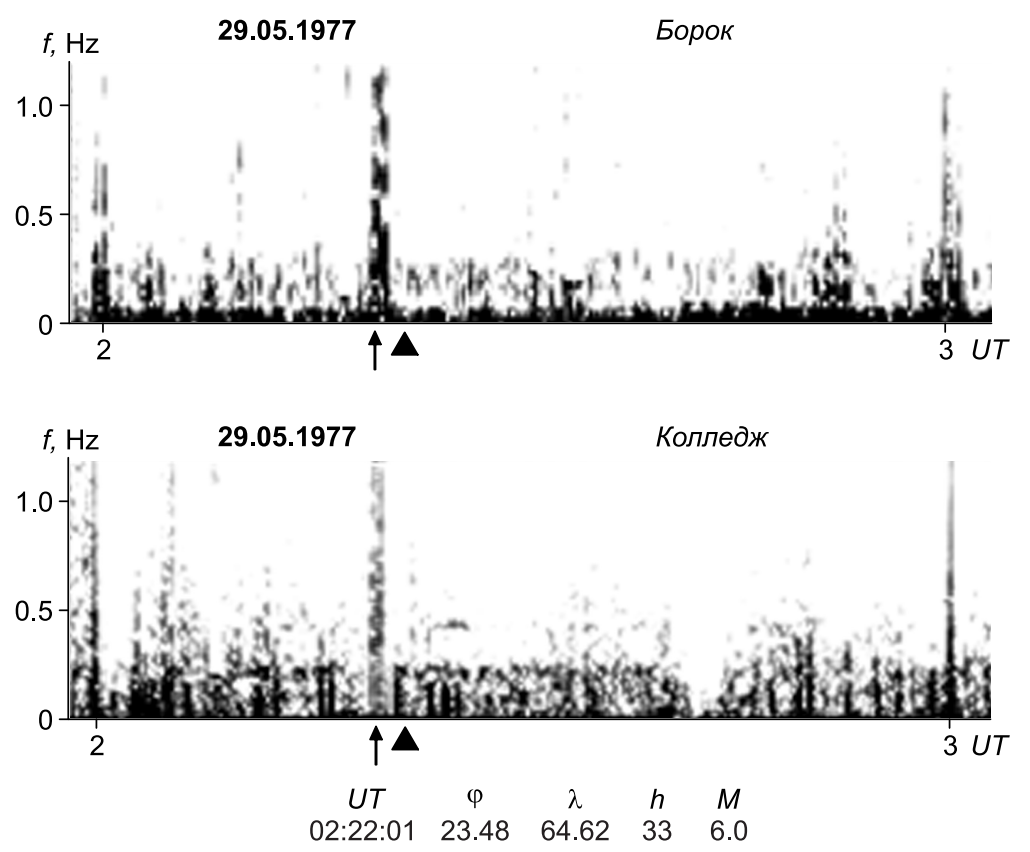

Рис. 5. Одновременное наблюдение сигнала-предвестника в разнесенных обсерваториях.

Fig. 5. Simultaneous observation of the precursor signal at the observatories located at large distances. 

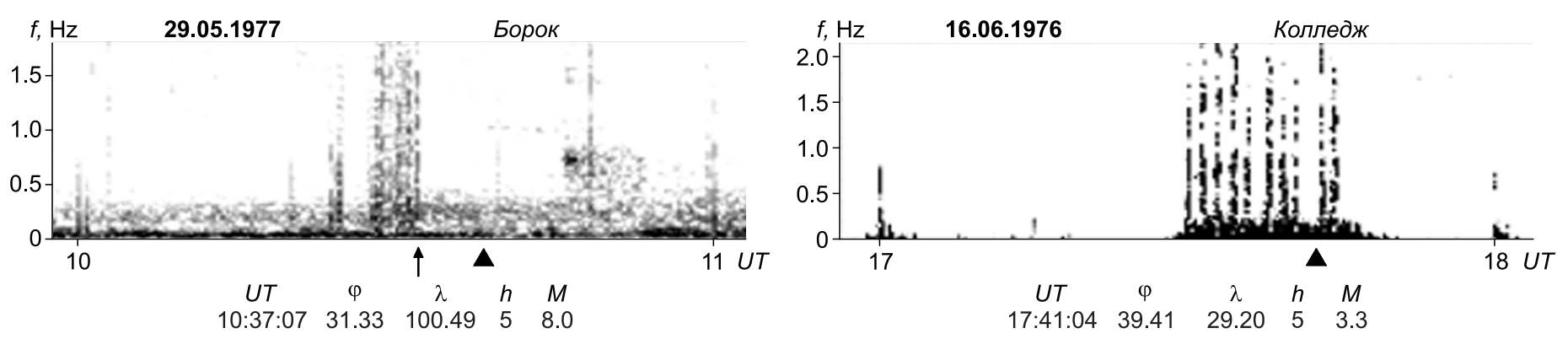

Рис. 6. Серии из следующих друг за другом импульсов, зарегистрированные перед землетрясениями по наблюдениям в обсерваториях «Борок» и «Колледж».

Fig. 6. Series of successive electromagnetic impulses recorded before the earthquakes. Data of the Borok and College observatories.

\section{4. ОБСУЖДЕНИЕ}

Итак, в рассмотренных нами случаях зарегистрированы сигналы, наблюдаемые в первые десятки секунд или минуты до землетрясения. По виду динамического спектра это были либо единичные, либо парные электромагнитные импульсы с дискретной структурой. Иногда наблюдались серии из следующих друг за другом импульсов. Во всех случаях сигналы имели подобные спектральные формы, один частотный диапазон и наблюдались в выделенном, относительно момента землетрясения, интервале времени. Попытаемся дать качественное объяснение полученным результатам.

Обнаруженные сигналы можно рассматривать как проявление механоэлектромагнитных преобразований в зоне землетрясений. Обычно при рассмотрении возможной причины генерации сейсмоэлектромагнитных сигналов предлагаются индукционный и пьезомагнитный механизмы [Guglielmi, Levshenko, 1996]. Но индукционный эффект предполагает резкие и заметные перемещения пород при импульсном излучении, что с большей вероятностью можно ожидать в момент землетрясения. В нашем эксперименте сигналы наблюдались до землетрясений, и их не было в момент главного толчка (см. рис. 1). По-видимому, процессы, протекающие в очаговой зоне, не могут рассматриваться в качестве источников электромагнитного излучения (впервые на эту особенность было обращено внимание в работе [Gokhberg et al., 1982]). Физика предвестников остается неясной. Возможно, объяснение следует искать в рамках модели Рейда, в которой землетрясение связывается с разрушением зацеплений на границе двух смежных плит [Reid, 1910]. Резкое сжатие пород, предшествующее их разрушению, приведет к генерации электромагнитного импульса (пьезомагнитный механизм) или серии из двух и более импульсов при неоднородной структуре межблочных зацеплений.
В рамках этой гипотезы находит объяснение и предпочтительное появление предвестников в выделенном и близком относительно момента землетрясения интервале времени.

Повторяемость предвестников можно рассматривать как повторяемость и подобие процессов, предваряющих происходящие в одном регионе землетрясения, с преобразованием механической энергии в энергию электромагнитного излучения. Тесная временная взаимосвязь электромагнитных и сейсмических явлений находится в хорошем соответствии с моделью землетрясений Рейда и не противоречит предлагаемому выше объяснению физической природы предвестников.

Дискретность спектра является типичной для случаев распространения пульсаций в ионосферном МГД-волноводе [Dovbnya et al., 2014]. Логично предположить, что для электромагнитных импульсов с дискретной структурой трассой их распространения от эпицентра землетрясения является ионосферный волновод. Канализируемые в таком волноводе сигналы способны распространяться вдоль земной поверхности с альвеновской скоростью 1000 км/с на значительные расстояния [Guglielmi, Troitskaya, 1973]. Разные условия на пути их следования могут привести к разной вероятности появления импульсов при одинаковой в среднем сейсмической активности.

\section{5. ЗАКЛЮЧЕНИЕ}

В своей работе мы стремились на экспериментальном материале показать реальность появления электромагнитных предвестников землетрясений. Результаты могут найти применение при поиске и разработке методов контроля за сейсмической активностью в потенциально опасных регионах. Наблюдение сигналов известной спектральной формы и их повторяемость дают принципиальную возмож- 
ность оперативного оповещения (за несколько минут) о предстоящем землетрясении.

\section{6. БЛАГОДАРНОСТИ}

Авторы благодарят А.С. Потапова и Б.И. Клайна за полезное обсуждение проблем, затронутых в работе. Работа выполнена в рамках базового финансирования программы ФНИ II.16 и поддержана Гос. темой 0144-2014-00116. Результаты частично получены с использованием ЦКП «Ангара» (http://ckp-rf.ru/ckp/3056/). Работа одного из авторов (А.Ю. Пашинина) поддержана РФФИ (проект № 19-05-00574).

\section{7. ЛИТЕРАTУPA / REFERENCES}

Adushkin V.V., Ryabova S.A., Spivak A.A., Kharlamov V.A., 2012. Response of the seismic background to geomagnetic variations. Doklady Earth Sciences 444 (1), 642-646. https://doi.org/10.1134/S1028334X12050157.

Bernardi A., Fraser-Smith A.C., McGill P.R., Villard O.G. Jr., 1991. ULF magnetic field measurements near the epicenter of the Ms 7.1 Loma Prieta earthquake. Physics of the Earth and Planetary Interiors 68 (1-2), 45-63. https://doi.org/ 10.1016/0031-9201(91)90006-4.

Dovbnya B.V., 2014. Electromagnetic precursors of earthquakes and their recurrence. Geofizicheskiy Zhurnal (Geophysical Journal) 36 (3), 160-165 (in Russian) [Довбня Б.В. Электромагнитные предвестники землетрясений и их повторяемость // Геофизический журнал. 2014. Т. 36. № 3. С. 160-165]. https://doi.org/10.24028/gzh.02033100.v36i3.2014.116069.

Dovbnya B.V., Potapov A.S., Guglielmi A.V., Rakhmatulin R.A., 2014. On influence of MHD resonators upon geomagnetic pulsations. Geofizicheskiy Zhurnal (Geophysical Journal) 36 (6), 143-152 (in Russian) [Довбня Б.В., Потапов А.C., Гульельми А.В., Рахматулин Р.А. О воздействии МГД резонаторов на геомагнитные пульсации // Геофизический журнал. 2014. Т. 36. № 6. С. 143-152]. https://doi.org/10.24028/gzh.0203-3100.v36i6.2014.111053.

Dovbnya B.V., Zotov O.D., Mostryukov A.O., Shchepetnov R.V., 2006. Electromagnetic signals close in time to earthquakes. Izvestiya, Physics of the Solid Earth 42 (8), 684-689. https://doi.org/10.1134/S1069351306080052.

Fraser-Smith A.C., Bernardi A., McGill P.R., Ladd M., Helliwell R.A., Villard O.G. Jr., 1990. Low-frequency magnetic field measurements near the epicenter of the Ms 7.1 Loma Prieta earthquake. Geophysical Research Letters 17 (9), 1465-1468. https://doi.org/10.1029/GL017i009p01465.

Gokhberg M.B., Morgounov V.A., Yoshino T., Tomizawa I., 1982. Experimental measurement of electromagnetic emissions possibly related to earthquakes in Japan. Journal of Geophysical Research: Solid Earth 87 (B9), 7824-7828. https://doi.org/10.1029/JB087iB09p07824.

Guglielmi A.V., 2007. Ultra-low-frequency electromagnetic waves in the Earth's crust and magnetosphere. PhysicsUspekhi 50 (12), 1197-1216. https://doi.org/10.1070/PU2007v050n12ABEH006413.

Guglielmi A.V., Levshenko V.T., 1996. Electromagnetic impulse from the earthquake source. Doklady AN 349 (5), 676-678 (in Russian) [Гульельми А.В., Левшенко В.T. Электромагнитный импульс из очага землетрясения // Доклады АН. 1996. Т. 349. № 5. С. 676-678].

Guglielmi A.V., Troitskaya V.A., 1973. Geomagnetic Impuslses and Diagnostics of the Magnetosphere. Nauka, Moscow, 208 p. (in Russian) [Гульельми А.В., Троицкая В.A. Геомагнитные пульсации и диагностика магнитосферы. М.: Наука, 1973. 208 с.].

Guglielmi A.V., Zotov O.D., 2012. Magnetic perturbations before the strong earthquakes. Izvestiya, Physics of the Solid Earth 48 (2), 171-173. https://doi.org/10.1134/S1069351312010065.

Hattori K., 2004. ULF geomagnetic changes associated with large earthquakes. Terrestrial Atmospheric and Oceanic Sciences 15 (3), 329-360.

Hayakawa M. (Ed.), 1999. Atmospheric and Ionospheric Electromagnetic Phenomena Associated with Earthquakes. Terra Scientific Publishing Company, Tokyo, $996 \mathrm{p}$.

Hayakawa M., 2001. Electromagnetic phenomena associated with earthquakes: review. IEEJ Transactions on Fundamentals and Materials 121 (10), 893-898. https://doi.org/10.1541/ieejfms1990.121.10_893.

Hayakawa M., Kawate R., Molchanov O.A., Yumoto K., 1996. Results of ultra-low-frequency magnetic field measurements during the Guam earthquake of 8 August 1993. Geophysical Research Letters 23 (3), 241-244. https:// doi.org/10.1029/95GL02863.

Hayakawa M., Molchanov O.A., 2002. Seismo-Electromagnetics Lithosphere-Atmosphere-Ionosphere Coupling. Terra Scientific Publishing Company, Tokyo, 477 p.

Ismaguilov V.S., Kopytenko Y.A., Hattori K., Voronov P.M., Molchanov O.A., Hayakawa M., 2001. ULF magnetic emissions connected with under sea bottom earthquakes. Natural Hazards and Earth System Sciences 1 (1-2), 23-31. https://doi.org/10.5194/nhess-1-23-2001. 
Kopytenko Y.A., Matiashvili T.G., Voronov P.M., Kopytenko E.A., Molchanov O.A., 1993. Detection of ultra-low-frequency emissions connected with the Spitak earthquake and its aftershock activity, based on geomagnetic pulsations data at Dusheti and Vardzia observatories. Physics of the Earth and Planetary Interiors 77 (1-2), 85-95. https://doi.org/ 10.1016/0031-9201(93)90035-8.

Kosterin N.A., Pilipenko V.A., Dmitriev E.M., 2015. On global ultralow frequency electromagnetic signals prior to earthquakes. Geofizicheskie Issledovaniya (Geophysical Research) 16 (1), 24-34 (in Russian) [Костерин H.A., Пилипенко В.А., Дмитриев Э.М. О глобальных ультранизкочастотных электромагнитных сигналах перед землетрясениями // Геофизические исследования. 2015. Т. 16. № 1. С. 24-34].

Liperovsky V.A., Pokhotelov O.A., Shalimov S.L., 1992. Ionospheric Precursors of Earthquakes. Nauka, Moscow, 304 p. (in Russian) [Липеровский В.A., Похотелов О.A., Шалимов С.Л. Ионосферные предвестники землетрясений. М.: Наука, 1992. 304 с.].

Molchanov O.A., 1990. Discovering of Ultra-Low-Frequency Emissions Connected with Spitak Earthquake and its Aftershock Activity on Data of Geomagnetic Pulsations Observations at Dusheti and Vardzija. Preprint No. 3 (888). IZMIRAN, Moscow, $27 \mathrm{p}$.

Molchanov O.A., Kopytenko Y.A., Voronov P.M., Kopytenko E.A., Matiashvili T.G., Fraser-Smith A.C., Bernardi A., 1992. Results of ULF magnetic field measurements near the epicenters of the Spitak (Ms=6.9) and Loma Prieta $(\mathrm{Ms}=7.1)$ earthquakes: Comparative analysis. Geophysical Research Letters 19 (14), 1495-1498. https://doi.org/10.1029/ 92GL01152.

Reid H.F., 1910. The Mechanics of the Earthquake, Vol. II. The California Earthquake of April 18, 1906: Report of the State Earthquake Investigation Commission. Carnegie Institution of Washington Publication No. 87. Carnegie Institution of Washington, Washington, D.C., 192 p.

Sobisevich L.E., Kanonidi K.K., Sobisevich A.L., 2010. Observations of ultra-low-frequency geomagnetic disturbances reflecting the processes of the preparation and development of tsunamigenic earthquakes. Doklady Earth Sciences 435 (2), 1627-1632. https://doi.org/10.1134/S1028334X10120160.

Sobisevich L.E., Sobisevich A.L., 2010. Dilatance structures and electromagnetic disturbances of the ULF range at the stages of preparation and development of a large seismic event. Bulletin of Earth Sciences Department of the Russian Academy of Sciences 2 (NZ6027), 202-213 (in Russian) [Собисевич Л.Е., Собисевич А.Л. Дилатансные структуры и электромагнитные возмущения УНЧ диапазона на этапах подготовки и развития крупного сейсмического события // Вестник Отделения наук о Земле РАН. 2010. Т. 2. NZ6027. C. 202-213]. https://doi.org/10.2205/2010NZ000045.

Sobolev G.A., 1993. Fundamentals of Earthquake Forecasting. Nauka, Moscow, 313 p. (in Russian) [Соболев Г.А. Основы прогнозирования землетрясений. М.: Наука, 1993. 313 с.].

Sobolev G.A., Zakrzhevskaya N.A., Kharin E.P., 2001. On the relation between seismicity and magnetic storms. Izvestiya, Physics of the Solid Earth 37 (11), 917-927.

Thomas J.N., Love J.J., Johnston M.J., 2009a. On the reported magnetic precursor of the 1989 Loma Prieta earthquake. Physics of the Earth and Planetary Interiors 173 (3-4), 207-215. https://doi.org/10.1016/j.pepi.2008.11.014.

Thomas J.N., Love J.J., Johnston M.J., Yumoto K., 2009b. On the reported magnetic precursor of the 1993 Guam earthquake. Geophysical Research Letters 36 (16), L16301. https://doi.org/10.1029/2009GL039020.

Zakrzhevskaya N.A., Sobolev G.A., 2002. On the seismicity effect of magnetic storms. Izvestiya, Physics of the Solid Earth 38 (4), 249-261.

Zakrzhevskaya N.A., Sobolev G.A., 2004. Influence of magnetic storms with a sudden start on seismicity in different regions. Vulkanologiya i Seismologiya (Journal of Volcanology and Seismology) (3), 63-75 (in Russian) [Закржевская Н.A., Соболев Г.А. Влияние магнитных бурь с внезапным началом на сейсмичность в различных регионах // Вулканология и сейсмология. 2004. № 3. С. 63-75].

\section{CВЕДЕНИЯ ОБ АВТOPAX | INFORMATION ABOUT AUTHORS}

Борис Викторович Довбня

канд. физ.-мат. наук, с.н.с.

Геофизическая обсерватория «Борок» Института физики Земли им. О.Ю. Шмидта РАН

152742, Ярославская область, Некоузский район, п. Борок, 142, Россия

\e-mail: dovbnya@inbox.ru

https://orcid.org/0000-0002-7019-5646
Boris V. Dovbnya

Candidate of Physics and Mathematics, Senior Researcher

Borok Geophysical Observatory of O.Yu. Schmidt Institute of Physics of the Earth of RAS

142, Borok 152742, Yaroslavl region, Nekouz district, Russia 


\section{Александр Юрьевич Пашинин}

ведущий инженер-электроник

Институт солнечно-земной физики СО РАН

664033, Иркутск, ул. Лермонтова, 126А, Россия

e-mail: pash@iszf.irk.ru

(iD) https://orcid.org/0000-0002-6795-7152

Равиль Анатольевич Рахматулин

докт. физ.-мат. наук, зав. обсерваторией

Институт солнечно-земной физики СО РАН

664033, Иркутск, ул. Лермонтова, 126А, Россия

e-mail: rav@iszf.irk.ru

(iD) https://orcid.org/0000-0001-6168-5291

\section{Aleksander Yu. Pashinin}

Lead Engineer

Institute of Solar-Terrestrial Physics, Siberian Branch of RAS

126A Lermontov street, Irkutsk 664033, Russia

\section{Ravil A. Rakhmatulin}

Doctor of Physics and Mathematics, Head of Observatory

Institute of Solar-Terrestrial Physics, Siberian Branch of RAS

126A Lermontov street, Irkutsk 664033, Russia 